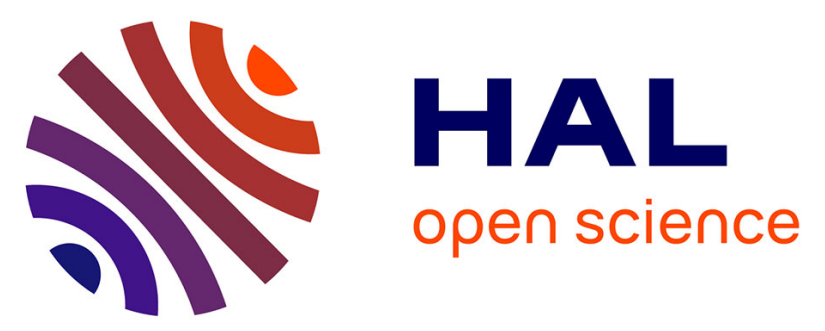

\title{
Population pharmacokinetics of mycophenolic acid in pediatric renal transplant patients using parametric and nonparametric approaches.
}

Aurélie Prémaud, Lt Weber, B. Tönshoff, V. W Armstrong, M. Oellerich, S. Urien, Pierre Marquet, Annick Rousseau

\section{To cite this version:}

Aurélie Prémaud, Lt Weber, B. Tönshoff, V. W Armstrong, M. Oellerich, et al.. Population pharmacokinetics of mycophenolic acid in pediatric renal transplant patients using parametric and nonparametric approaches.. Pharmacological Research, 2011, 63 (3), pp.216-224. 10.1016/j.phrs.2010.10.017 . inserm-00541907

\section{HAL Id: inserm-00541907 https://www.hal.inserm.fr/inserm-00541907}

Submitted on 30 Mar 2012

HAL is a multi-disciplinary open access archive for the deposit and dissemination of scientific research documents, whether they are published or not. The documents may come from teaching and research institutions in France or abroad, or from public or private research centers.
L'archive ouverte pluridisciplinaire HAL, est destinée au dépôt et à la diffusion de documents scientifiques de niveau recherche, publiés ou non, émanant des établissements d'enseignement et de recherche français ou étrangers, des laboratoires publics ou privés. 


\title{
Population pharmacokinetics of mycophenolic acid in pediatric renal transplant patients using parametric and nonparametric approaches
}

\author{
A Prémaud ${ }^{1,2}$, LT Weber ${ }^{3}$, B Tönshoff ${ }^{4}$, V W Armstrong ${ }^{5}$, M Oellerich ${ }^{5}$, S Urien ${ }^{6}$, P Marquet \\ ${ }^{1,2,7}$, A Rousseau ${ }^{1,8}$ \\ ${ }^{1}$ INSERM U850, Limoges, France \\ ${ }^{2}$ Univ Limoges, Faculty of Medicine, Laboratory of Clinical Pharmacology, Limoges, France \\ ${ }^{3}$ Pediatric Nephrology, University Children's Hospital, Ludwig-Maximilian's-University, Munich, \\ Germany \\ ${ }^{4}$ University Children's Hospital, 69120 Heidelberg, Germany \\ ${ }^{5}$ Department of Clinical Chemistry, University Medical Center of Göttingen, Germany \\ ${ }^{6}$ CIC-0901 - INSERM \& EA-3620 - Université Paris Descartes, France \\ ${ }^{7} \mathrm{CHU}$ Limoges, Department of Pharmacology and Toxicology, Limoges, France \\ ${ }^{8}$ Univ Limoges, Faculty of Pharmacy, Department of Biomathematics, Limoges, France \\ Corresponding author: \\ Dr. Annick Rousseau, \\ INSERM U850, Laboratory of Clinical Pharmacology \\ Faculty of Medicine, Limoges University \\ 2 rue du Dr. Marcland \\ 87025 Limoges cedex France \\ Tél. +33 $555435846 \quad$ Fax. +33555435936 E-mail: annick.rousseau@unilim.fr
}




\section{ABSTRACT}

Mycophenolic acid (MPA) is an immunosuppressive drug widely used in the prevention of acute rejection in pediatric renal transplant recipients and is characterized by a wide inter-individual variability in its pharmacokinetics. The aim of this study was to compare population pharmacokinetic modeling of MPA in pediatric renal transplant recipients given mycophenolate mofetil, the ester prodrug of MPA, using parametric and nonparametric population methods. The data from 34 pediatric renal transplants (73 full pharmacokinetic profiles obtained on day 21, months 3,6 and 9 posttransplant) were analyzed using both the nonlinear mixed-effect modeling (NONMEM) and nonparametric adaptive grid (NPAG) approaches, based on a two-compartment model with first order lagged time absorption and first order elimination. The predictive performance of the two models was evaluated in a separate group of 32 patients. Higher mean population parameter values and ranges of individual pharmacokinetic parameters were obtained with NPAG, especially for the elimination constant ke: mean $1.16 \mathrm{~h}^{-1}\left(0.26\right.$ to $\left.4.33 \mathrm{~h}^{-1}\right)$ and $0.78 \mathrm{~h}^{-1}\left(0.66\right.$ to $\left.1.15 \mathrm{~h}^{-1}\right)$ with NPAG and NONMEM, respectively. With NPAG, the skewness and kurtosis values for ke (2.03 and 7.80, respectively) were far from the theoretical values expected for normal distributions. Such a non-normal distribution could explain the high value of shrinkage (35\%) obtained for this parameter with the parametric NONMEM method. Bayesian forecasting of mycophenolic acid exposure using the NPAG population pharmacokinetic parameters as priors yielded a better predictive performance, with a significantly smaller bias than with the NONMEM model $(-1.68 \%$ vs $-9.53 \%, p<0.0001)$. In conclusion, in the present study, NPAG was found to be the most adequate population pharmacokinetic method to describe the pharmacokinetics of MPA in pediatric renal transplant recipients.

Keywords: mycophenolic acid, pediatric, renal transplantation, population pharmacokinetics, parametric, nonparametric. 


\section{INTRODUCTION}

Mycophenolate mofetil (MMF), the ester prodrug of mycophenolic acid (MPA) has been approved for immunosuppressive therapy in combination with calcineurin inhibitors following renal transplantation, both in adults and children. MMF, which is dosed based on body surface area in the pediatric population, was shown to be effective in the prevention of acute rejection in pediatric renal transplant recipients, and is now widely used for maintenance immunosuppressive therapy in this population [1$4]$.

As in adults, the pharmacokinetics (PK) of MPA in pediatric renal transplant recipients is characterized by a large inter- and intra-individual variability, particularly in the early post-transplant period $[5,6]$. This variability is clinically relevant as shown by the association between the risk of acute rejection episodes and MPA AUC ${ }_{0-12 h}$ values [5,7]. The pharmacokinetics of MPA is also characterized by a large inter-individual variability and a gradual increase in dose-normalized exposure over time in the first three months post-transplantation [6,8]. In a population analysis based on a two-compartment model with time-lagged first order absorption, van Hest et al [9] found that this time dependency could be due to a combination of improving creatinine clearance, progressively increasing albumin, hemoglobin and decreasing cyclosporine predose concentrations (C0) during the first 6 months after transplantation.

A decreased incidence of acute rejection has been found as a result of therapeutic drug monitoring (TDM) of MPA in adult [10] renal allograft recipients, and suggested in pediatric [11] recipients. However, establishing such a strategy requires characterization and modeling of MPA pharmacokinetics in the target population. Sophisticated pharmacokinetic models able to describe the complex plasma concentration profiles observed in adult allograft recipients in the early posttransplantation period [12], or the enterohepatic recirculation often observed when MMF is combined with tacrolimus [13-16] or sirolimus [17], have been reported.

Some authors have proposed algorithms based on a limited number of sampling time points, able to predict MPA inter-dose area under the curve $\left(\mathrm{AUC}_{0-12 h}\right)$ in pediatric kidney graft recipients on MMF and cyclosporine therapy [18-20]. Bayesian forecasting methods based on population PK models represent an alternative approach to predict the individual MPA PK parameters, hence concentration profiles. Using Bayesian estimators developed in populations of adult renal transplants at early and late post-transplantation periods, and based on a limited strategy of three time points $(20 \mathrm{~min}, 1 \mathrm{~h}$ and 
3h post-dose) [21], the APOMYGRE trial showed the feasibility, efficacy, safety and cost-effectiveness of MMF dose individualization in adult renal transplant patients using such Bayesian estimators $[10,22,23]$. However, these Bayesian estimators were developed using the iterative two-stage Bayesian method and an in-house computer program. A more common approach is one-stage population pharmacokinetic modeling. Payen et al. [24] reported a population pharmacokinetic analysis of MPA in pediatric and also adolescent renal transplant patients performed using NONMEM (NONlinear Mixed-Effect Modeling software), and the development of a Bayesian estimator based on this population model to predict MPA exposure. Most of the pharmacokinetic profiles were collected in stable patients as more than half of the patients were grafted for more than six months.

To the best of our knowledge, the published one-stage population pharmacokinetic analyses of MPA were based on a parametric approach, mostly using NONMEM [13, 15, 24- 29]. Usually, following such a parametric approach the influence of covariates on the PK parameters is analyzed and the remaining, unexplained inter-individual variability of the PK parameters is assumed to follow a predefined distribution e.g. a normal or log-normal distribution. However, when such a parametric approach is inappropriate to describe the unexplained inter-individual variability, implementation of Box-Cox or Logit transformations in NONMEM have been recently proposed [30]. These methods have been called "semi-parametric" as the shape of the transformed distribution is no more Gaussian. Non-parametric methods can describe the actual distribution of random effects whatever its shape [3133]. These methods are able to directly discover, in the population itself, unsuspected subpopulations of individuals (i.e., without external help such as descriptors, covariates or individual Bayesian posterior parameter estimates), which is not possible with parametric techniques. Non-parametric methods also provide the traditional estimates of the parameter: mean, standard deviation, mode, median, correlation and covariance.

The aim of this study was to characterize MPA PK in pediatric renal transplant patients using several population approaches, namely nonlinear mixed-effect modeling (NONMEM) with or without semiparametric transformations of the inter-individual variability, and the nonparametric adaptive grid (NPAG) method.

\section{MATERIAL AND METHODS}




\subsection{Patients}

The population analysis was performed using pharmacokinetic data issued from two different cohorts of pediatric renal transplant recipients on a comparable immunosuppressive regimen consisting of mycophenolate mofetil $\left(600 \mathrm{mg} / \mathrm{m}^{2}\right.$ body surface area twice a day, up to a maximum of $2 \mathrm{~g} /$ day), cyclosporine and corticosteroids. The two patient cohorts have been described in detail in a previous paper [18]. 44 patients (28 boys) of the Cohort of the German Study group on MMF therapy, with a median age of 10.7 years (range, 3.2 to 15.9) and 22 patients (14 boys) of the Tricontinental MMF suspension trial, with a median age of 7 years (range, 1 to 16) were analyzed. The parents of the patients gave informed consent for the participation of their children and the clinical trial were designed in accordance with legal requirements. One to three profiles were collected in each patient, so that the 66 pediatric patients provided 129 full pharmacokinetic profiles (of 9 blood samples collected at: predose, $20 \mathrm{~min}, 40 \mathrm{~min}, 75 \mathrm{~min}, 2,4,6,8$ and 12h after dosing; or of 8 blood samples collected at: predose, $30 \min 1,1.5,2,4,8$ and $12 \mathrm{~h}$ after dosing) on day 21 and months 3,6 , and 9 posttransplant.

The whole dataset was randomly divided into 2 groups: a reference group made of 34 patients (73 pharmacokinetic profiles) was used to develop the population pharmacokinetic models and a test group of the remaining 32 patients (56 pharmacokinetic profiles) was used to evaluate the predictive performance of the population models.

\subsection{MPA analysis}

MPA plasma concentrations were measured using a reverse-phase high-performance liquid chromatography method with ultraviolet detection previously described in details [34]. The error pattern of the assay was determined by measuring several assay samples (which cover the full working range of the assay) in at least quadruplicate leading to find standard deviation (SD) for each of the samples. Then, the overall relationship between the plasma concentration and the SD with which it has been measured was expressed by the following polynomial equation:

$S D=0.963+0.0227 C+0.008 C^{2}$ where $\mathrm{SD}$ is the assay standard deviation and $\mathrm{C}$ is the measured plasma concentration.

\subsection{Pharmacokinetic population modeling}


The plasma concentration versus time data of the index group were analyzed using two population pharmacokinetic approaches in parallel: NONMEM and nonparametric adaptive grid (NPAG) methods.

\subsubsection{NONMEM analysis}

Data were analyzed using NONMEM version $\mathrm{VI}[35]$ and Wings for NONMEM®) version 614 (N. Holford, Auckland, New Zealand). All population pharmacokinetic analyses were performed using the first order conditional estimation (FOCE) method with interaction.

A two-compartment model with first-order, lagged time absorption and first-order elimination best described the data.

Unexplained inter-individual variability (IIV) was initially modeled following an exponential error model. The semi-parametric approach proposed by Petersson et al. [30] was also tested: two transformations with estimated shape parameter (Box-cox and logit) were explored to evaluate if they could improve data fitting.

Inter-occasion variability (IOV) was modeled using an exponential error model. Diagonal matrix and full variance/covariance matrix (using the Block() command) was successively tested to estimate interpatients random variabilities (ETAs). The variance/covariance matrix was also tested for certain parameters only, if appropriate. Additive, proportional and combined (i.e., additive and proportional) error models were tested to describe the residual variability.

The influence of gender, age and weight (the only covariates available) on the absorption and elimination parameters and on the apparent central volume of distribution was investigated. Covariates selected to enter the intermediate model simultaneously had to lead individually to a significant $(p<0.01)$ decrease in the objective function from the covariate-free model. Finally, in a backwarddeletion strategy, each covariate was independently removed from the full model to confirm its relevance. If the increase in the objective function was not statistically significant $(p<0.001)$, the relationship between this covariate and the respective pharmacokinetic parameter was not taken into account in the final model. The final model thus only included the significant covariates. At this stage, the clinical relevance of the covariate(s) was also appraised taking into account the reduction in IIV. Potential over-parameterization was tested taking into account the condition number (calculated as the square root of the ratio of the smallest to the largest eigenvalue) and the extreme eigenvalues. The stability and performance of the final population model were evaluated using the bootstrap approach. 
Thousand bootstrap sets were drawn by resampling from the original dataset, each providing population PK parameter estimates. The mean, median and 95\% confidence interval values estimated from the 1,000 bootstrap sets for each PK parameter were compared to those obtained with the original dataset.

The adequacy of the final population model was evaluated by a Visual Predictive Check (VPC) performed using the ' $R$ for NONMEM' package [36]. A total of 1,000 datasets were simulated from the final model using the original data set. Plots of the median and $90 \%$ confidence interval of the simulated concentration versus time profiles were generated to check that the distribution of the observed concentration-time profiles was reasonably contained within this confidence interval. Because the MMF dose (in $\mathrm{mg}$ ) was different in each patient, the predictions and the observations were dose normalized on the basis of the mean dose administered in the reference group (thereby the simulations were done using any dose). Moreover, the extent of shrinkage on each parameter ( $\eta$ shrinkage) and on individual predictions ( $\varepsilon$-shrinkage) was computed following Karlsson and Savic recommendations [37]. $\eta$-shrinkage was calculated using the following formula : $1-\operatorname{SD}\left(\eta_{\text {parameter }}\right) / \omega$, where $\operatorname{SD}\left(\eta_{\text {parameter }}\right)$ is the standard deviation of individual estimates of $\eta$ for one parameter and $\omega$ the population estimated standard deviation of the corresponding random effect. Large values of shrinkage (higher than 20-30\%) would generally be associated with poor individual estimates of that parameter. $\varepsilon$-shrinkage was calculated as 1-SD (IWRES), where IWRES are the individual weighted residuals, and will thus increase from zero toward one as data become less informative.

\subsubsection{NPAG analysis}

The nonparametric population analysis was performed using BigWinPops version 0.03 (R. Jeliffe, Laboratory of Applied Pharmacokinetics, USC Keck School of Medicine, Los Angeles, USA). One- and two-compartment models with first-order elimination and first-order absorption with or without a lagtime were tested. The best model was selected on the basis of the log-likelihood of the results.

The iterative two-stage Bayesian (IT2B) method was first used to obtain initial estimates of the PK parameter ranges. Then, nonparametric population analysis was performed using the NPAG program (29]. Combined with an interior point rather than an expectation-maximization algorithm, NPAG constitutes an improved version of the Nonparametric Expectation Maximization program, which computes the discrete joint probability density functions of the population pharmacokinetic parameters. 
NPAG also computes a so-called $\gamma$ parameter, representing an overall measure of all the other sources of intra-individual variability besides the assay error, such as the errors in preparation and administration of the doses, errors in times of administration, misspecification of the pharmacokinetic model (residual error). Gamma is used as a multiplier of the assay error polynomial (Total error SD $=$ gamma $\times$ Assay standard deviation). A value of gamma of 1.0 suggests that there is no other source of variability than the assay. A value of gamma $>1.0$ suggests that the environmental noise needs to be taken into account in the overall error by multiplying the value of gamma by the analytical error pattern.

\subsubsection{Evaluation of the performance of the population models}

The performance of NONMEM or NPAG population models was evaluated both in the index and in the test patient groups using Bayesian forecasting.

Individual Bayesian estimates of individual pharmacokinetic parameters and AUCs were calculated for each patient included in the index group, using as priors the mean values and variability of the population pharmacokinetic parameters previously obtained with the best models retained with NONMEM and NPAG, respectively. Goodness of fit of the population models was evaluated by comparing Bayesian estimated and observed MPA plasma concentrations.

Individual Bayesian estimates of MPA exposure and concentration values were also calculated for the 36 patients included in the test group (56 concentration-time profiles) using the population characteristics of the index group and all the concentration-time points available.

With NONMEM, the individual Bayesian estimates were obtained using the POSTHOC Bayesian option. With NPAG, Bayesian estimation of individual parameter values in independent patients was not available. Consequently, the final population model (i.e., mean parameter vector and full covariance matrix) was inserted into the PRIOR subroutine of the ADAPT II software package [38], to allow for maximum a posteriori Bayesian estimation. Two hypotheses related to the prior distribution model (normal or lognormal) were tested.

\subsection{Statistical analyses}

All statistical analyses were performed using the $R$ software [39]. 
Comparisons of patients' characteristics and MPA exposure indices between the index and test groups were performed using the Student t-test.

Estimated and observed MPA concentrations and AUCs were compared using the mean relative error (MRE\%) as a measure of bias, and root mean squared prediction error (RMSE) as a measure of precision. Statistical significance of the MRE was evaluated using the paired t-test.

\section{RESULTS}

\subsection{Data description}

The characteristics of the patients included in the reference and test groups are summarized in Table 1. No significant differences were observed between the two groups. Figure 1 shows the individual concentration-time curves observed during the four post-transplantation periods studied. For some profiles (less than 10 out of 129) a second concentration peak was observed at approximately $6 \mathrm{~h}$ postdose, but most profiles exhibited a single concentration peak. The dose-normalized MPA AUC ${ }_{0-12}$ [(AUC/dose $)]$, calculated using the linear trapezoidal rule, was not significantly different between the two groups $(p=0.126)$. A wide distribution of these dose-normalized MPA AUC $\mathrm{C}_{0-12}$ was observed in each group at each period (Table 1), with some extremely high values, i.e. a very low apparent clearance. The Shapiro-Wilk normality test showed a significant deviation from the normal distribution of $A \cup C /$ dose in both the reference $(p<0.001)$ and the test $(p=0.0018)$ groups, reflecting a non Gaussian distribution of the apparent clearance.

\subsection{Population modeling}

\subsubsection{NONMEM analysis}

A two-compartment model with first-order, lagged time absorption and first-order elimination adequately described the concentration data. The model parameters were the absorption rate constant $(\mathrm{ka})$, the apparent central volume of distribution $(\mathrm{Vc} / \mathrm{F})$, the rate constant from central to peripheral compartments $(k 12)$, the rate constant from peripheral to central compartments (k21), the elimination rate constant (ke) and the absorption lag-time. Introduction of IIV on ka, Vc/F, k12, ke and lag-time significantly improved the fit of the model. As the Box-cox and logit transformations failed to 
improve model fitting and shrinkage, the IIV was modeled using an exponential error model. Inclusion of IOV on ka and Vc/F led to a significant decrease of the objective function value, but to negligible IIV values $(1.04 \mathrm{E}-5 \%$ and $0.17 \%$, respectively). Consequently, the final model included IOV on ka and $\mathrm{Vc} / \mathrm{F}$, IIV on k12, ke and lag-time, and a combined, additive and proportional, residual error. Finally, a simple diagonal matrix was selected. The population pharmacokinetic parameters (popPK) obtained are summarized in Table 2.

Among the covariates investigated, only the post-transplantation period influenced ke with a statistically significant decrease of the objective function of 12 units $(p<0.01)$. However, introduction of this covariate led to a high value of shrinkage estimates for ke (40\%) and did not seem to significantly reduce ke unexplained inter-individual variability that still reached $14.3 \%$ (Cl95\%: $0-27.4 \%)$ versus 19.9\% (Cl95\%: 0 - 31.5\%) with the covariate-free model. Therefore, the covariate-free model was finally retained. Diagnostic plots are shown in figure 2. The scatter plot of predicted (PRED) and individual model-predicted concentrations (IPRED) versus observed concentrations (DV) showed no major bias (Figure 2), while $91 \%$ of weighted residuals (WRES) were in the $(-2 ;+2)$ range.

Estimates of the shrinkage for ETAs of ka, k12, ke, Vc/F and lag-time were 0.096, 0.093, 0.352, 0.067 and 0.199 , respectively. The condition number was 14.7 . The residual error was fairly high $(38.7 \%$ and $0.08 \mathrm{mg} / \mathrm{L}$ for the proportional and additive parts, respectively), but not shrunk (0.124). The mean parameter estimates obtained using the bootstrap procedure (1000 runs) were similar to those previously obtained with the original dataset (Table 2).

Figure 3 shows the results of the VPC for the final model. Simulations were based on dose-normalized concentrations, using the mean MMF dose of $625 \mathrm{mg}$ observed in the index group. The results are presented separately for profiles observed at early and stable post-transplant periods. The observations were within the $90 \%$ confidence interval but not distributed symmetrically relative to the median prediction. This shows the tendency of the model to underestimation.

\subsubsection{NPAG analysis}

The two-compartment model with first-order, time-lagged absorption and first-order elimination provided the best description of the data. The pharmacokinetic model included the same 6 pharmacokinetic parameters as previously described for the parametric analysis: ka, Vc/F, k12, k21, ke and lag-time. 
The initial IT2B analysis led to a value of $\mathrm{y}$ of 0.89 suggesting that there was no other source of variability than the assay SD to consider in the model. Consequently, $y$ was set to 1 , meaning that the total error (both assay and environmental) SD was equal to the error assay pattern SD.

MPA pharmacokinetic parameter estimates in the index group are summarized in Table 3. Mean and median values of ka, lag-time and k21 were far apart, showing the heterogeneity and the asymmetry of their distribution. The results of the NPAG predictive performance evaluation are shown in Figure 4. The scatter plot of observed concentrations versus individual plasma concentrations predicted using the median parameter values of each patient's nonparametric Bayesian joint density (Bayesian posterior predicted levels) showed no major bias.

\subsubsection{Comparison between NONMEM and NPAG population models}

Except for $\mathrm{V} c / F$, the mean population pharmacokinetic parameter values obtained with NPAG were higher by at least $50 \%$ than with NONMEM. The two methods led to a similar high value of ka in an "outlier" patient, characterized by a very fast absorption of the drug. The range of the individual ke and lag-time values was much narrower with NONMEM ( 0.66 to $1.15 \mathrm{~h}^{-1}$ vs. 0.26 to $4.33 \mathrm{~h}^{-1}$ and 0.21 to $0.33 \mathrm{~h}$ vs. 0 to $1.85 \mathrm{~h}$, respectively). Interestingly, the ke and lag-time skewness (2.03 and 1.70 ) and kurtosis (7.80 and 5.38) were not in favour of a normal distribution for these parameters.

\subsection{Evaluation of the predictive performance of the population models in the test group}

The predictive performance of the two final population models developed according to the NONMEM and NPAG methodologies is reported in Tables 2 and 3. The NPAG model resulted in more accurate and precise prediction of MPA AUC and concentrations than the NONMEM model, as shown by the lower and non significant bias and RMSE values.

Using the nonparametric model, the best results were obtained with the lognormal prior distribution model option in ADAPT II. This model provided significantly lower mean AUC bias than the parametric model on M3 $(0.79 \%$ vs $-12.34 \%, \mathrm{p}<0.001), \mathrm{M} 6(7.50 \%$ vs $-11.35 \%, \mathrm{p}<0.05)$ and $\mathrm{M} 9(-0.25 \%$ vs $12.39 \%, \mathrm{p}<0.001)$ but not on W3 $(3.56 \%$ vs $2.97 \%$, NS).

\section{DISCUSSION}


To the best of our knowledge, this study is the first to report population pharmacokinetic analysis of MPA using both parametric and nonparametric approaches (NONMEM and NPAG methods, respectively) in renal transplant children (from 1 to 18 years) and to show the superiority of the nonparametric modeling method.

All the previous MPA population pharmacokinetic models developed both in adults and in children were based on parametric methods. Two population pharmacokinetic studies in pediatric renal transplant recipients were previously reported [24, 40]. Unlike the current study, Payen et al. [24], analyzed data collected in children and young adults, mainly in the stable post-transplantation period. Recently, Zhao et al. [40] reported a population pharmacokinetic model developed in children mainly focused on the period from before dosing to $3 \mathrm{~h}$ after ( $91 \%$ of the samples collected within this period). However, this sampling design did not allow describing the time-concentration curves beyond three hours post-dosing, i.e. when the MPA PK profiles are the most complex and probably the most variable. Therefore, different modeling strategies have been proposed in adults: (i) structural sophisticated models allowing to describe profile with two concentration peaks [41]; (ii) enterohepatic circulation [13,17] partly inhibited when cyclosporine is co-administered [42,43]; (ii) time-dependent clearance $[15,9]$. These approaches were tested herein and failed to improve the data fitting. In adult patients, the MPA pharmacokinetic modeling studies investigating the first weeks post-transplantation failed to estimate with precision the concentration profiles or yielded very high residual variability [15, $26,27]$.

In the present study, the observed MPA concentration-time profiles mostly exhibited a single peak concentration and were best described by a two-compartment model with time-lagged first-order absorption. This is consistent with previous reports where MMF was co-administered with cyclosporine $[15,17,24,25,27]$. The analysis performed with NPAG led to higher values of MPA population PK parameters (except for $\mathrm{Vc} / \mathrm{F}$ ) and to a wider inter-individual variability than with NONMEM (especially for the elimination constant). The differences observed between the two approaches can be explained by the different calculation methods used by NONMEM and NPAG. Indeed, NONMEM is a parametric method in that it assumes a normal or log-normal distribution of the PK parameters and tends to put less weight on extreme values. This tendency can be highlighted by the $\eta$-shrinkage expressing the shrinkage of the variance of empirical Bayes estimates distribution toward zero. 
The high value of the calculated ke $\eta$-shrinkage (35\%), illustrates the trend to estimate individual ke values close to the population mean ( $\eta$ expressing the deviation of the individual parameter estimates from the typical parameter value in the population) which may be a problem since the estimates of this parameter are used for TDM. Indeed, with such high shrinkage values, posthoc $\eta_{s}$ cannot be considered as informative about the true $\eta$ distribution and the individual Bayesian estimates would be obtained with a large imprecision, which is hardly compatible with the development of Bayesian estimators for TDM. This result could be expected as the initial non-compartmental analysis showed a non-Gaussian distribution of the dose-normalized AUC in the reference group.

One consequence of $\eta$-shrinkage is that covariate relationships may be hidden, falsely induced, or the shape of the true relationship distorted [37]. This may partly contribute to the fact that, in the present study, no covariate was found significant. However, very few potential covariates were registered in the database used.

The high value of shrinkage obtained for the elimination constant rate prompted us to ask ourselves about the relevance of standard parametric modeling (using fixed exponential transformation) and its ability to describe the shapes of the parameter distribution. Thus, two semi-parametric transformations (i.e. Box-cox and logit transformations) [30] were investigated but failed to improve the model fit. Finally, a nonparametric approach was used. In NPAG, no assumption is made about the shape of the parameters distribution which allows for many possible distributions and enables detection of the full diversity in the distribution of population parameters. This approach could reveal the true nature of the distribution of the elimination rate constant ke, allowing the description of subpopulations and the extreme values [44]. However, it is not possible (in the current state of the art) to determine the inaccuracy of estimates obtained using such a nonparametric approach. Consequently, shrinkage cannot be calculated.

This study performed in children is the first focusing on a non normal distribution of MPA PK parameters. Such deviation from normality was not reported in adults. The high heterogeneity of the elimination constant values in children suggests the requirement of a wide range of doses to reach the target exposure. In clinical practice, this could result in a higher variability of administered doses in children than in adults. The mechanisms underlying this age-related difference in MMF dose requirements are largely unknown but could be related to developmental changes (also known as 
ontogeny of drug disposition) of the UDP-glucuronosyltransferases, the enzyme family mainly responsible for MPA metabolism [40].

The results of the Bayesian estimation of MPA AUC and concentrations in the test patient group showed that the NPAG had a better predictive performance than the NONMEM model, as it yielded smaller and non-significant bias. The biased estimates obtained with the NONMEM model in the present case make it unsuitable for TDM. This result supports the assumption that NPAG would be preferable to NONMEM in case of multimodal or non-normal distribution of any one of the PK parameters in the model. However, the limit of the present results lies in the fact that Bayesian estimation of individual parameter values in the independent group of patients was not feasible in BigWinPops and was performed using ADAPT II, i.e. under the usual normality assumptions, which is not optimal in the case of non-normally distributed PK parameters.

Another limitation is the lack of relevant covariates in our database. Indeed, while few demographic covariates, available for all patients, could be included in the population analysis, no major clinical variables, such as renal function or serum albumin which have been previously found related to MPA exposure in adults [13], were investigated due to many missing data. This is not a barrier to nonparametric analysis as, due to no assumption about the shape of the parameter distributions, unsuspected subpopulations of subjects can be detected without additional aid from covariates or other descriptors [32,44]. Finally, the population model analysis reported in the present study dealt with pediatric renal transplant patients receiving a MMF/ciclosporine regimen and cannot be extrapolated to another immunosuppressive regimen like the MMF/tacrolimus association which is becoming more common in prevention of acute rejection.

In conclusion, NPAG was a more adequate population pharmacokinetic method to describe the pharmacokinetics of MPA in pediatric renal transplant recipients. This finding could be linked to the studied database which included very few covariates able to explain the pharmacokinetic variability of MPA (such as serum albumin, blood hemoglobin and cyclosporine concentrations). 


\section{ACKNOWLEDGEMENTS}

The authors thank R Jelliffe R, M Van Guilder and A Ghandi (Laboratory of Applied Pharmacokinetics, School of Medicine, University of Southern California, Los Angeles, California) for their advice and their valuable methodological assistance for nonparametric analyses. 


\section{REFERENCES}

[1] Hocker B, Weber LT, Buncman T, Rashford M, Tonshoff B. Mycophenolate mofetil suspension in pediatric renal transplantation: three-year data from the tricontinental trial. Pediatr Transplant 2005; 504-11.

[2] Jungraithmayr T, Staskewitz A, Kirste G, Böswald M, Bulla M, Burghard R, Dippell J, Greiner C, Helmchen U, Klare B, Klaus G, Leichter HE, Mihatsch MJ, Michalk DV, Misselwitz J, Plank C, Querfeld U, Weber LT, Wiesel M, Tönshoff B, ZimmerhackI LB; For the German Pediatric Renal Transplantation Study Group. Pediatric renal transplantation with mycophenolate mofetil-based immunosuppression without induction: results after three years. Transplantation 2003; 75: 454-61.

[3] Bunchman T, Navarro M, Broyer M, Sherbotie J, Chavers B, Tönshoff B, Birk P, Lerner G, Lirenman D, Greenbaum L, Walker R, ZimmerhackI LB, Blowey D, Clark G, Ettenger R, Arterburn S, Klamerus K, Fong A, Tang H, Thomas S, Ramos E. The use of mycophenolate mofetil suspension in pediatric renal allograft recipients. Pediatr Nephrol 2001; 16: 978-84.

[4] Staskewitz A, Kirste G, Tonshoff B, Weber LT, Boswald M, Burghard R, Helmchen U, Brandis M, Zimmerhackl LB. Mycophenolate mofetil in pediatric renal transplantation without induction therapy: results after 12 months of treatment. German Pediatric Renal Transplantation Study Group. Transplantation 2001; 71: 638-44.

[5] Weber LT, Shipkova M, Armstrong VW, Wagner N, Schütz E, Mehls O, ZimmerhackI LB, Oellerich M, Tönshoff B. The pharmacokinetic-pharmacodynamic relationship for total and free mycophenolic Acid in pediatric renal transplant recipients: a report of the german study group on mycophenolate mofetil therapy. J Am Soc Nephrol 2002; 13:759-68.

[6] Weber LT, Lamersdorf T, Shipkova M, Niedmann PD, Wiesel M, Zimmerhackl LB, Staskewitz A, Schütz E, Mehls O, Oellerich M, Armstrong VW, Tönshoff B. Area under the plasma concentrationtime curve for total, but not for free, mycophenolic acid increases in the stable phase after renal transplantation: a longitudinal study in pediatric patients. German Study Group on Mycophenolate Mofetil Therapy in Pediatric Renal Transplant Recipients. Ther Drug Monit 1999; 21:498-506. 
[7] Weber LT, Shipkova M, Armstrong VW, Wagner N, Schütz E, Mehls O, ZimmerhackI LB, Oellerich $M, T o ̈ n s h o f f ~ B$. The pharmacokinetic relationship for total and free mycophenolic acid in pediatric renal transplant recipients: a report of the German Study Group on mycophenolate mofetil therapy. J Am Soc Nephrol 2002; 13:759-68.

[8] Weber LT, Hoecker B, Armstrong VW, Oellerich M, Tönshoff B. Long-term pharmacokinetics of mycophenolic acid in pediatric renal transplant recipients over 3 years posttransplant. Ther Drug Monit 2008;30: 570-5.

[9] van Hest RM, van Gelder T, Bouw R, Goggin T, Gordon R, Mamelok RD, Mathot RA. Timedependent clearance of mycophenolic acid in renal transplant recipients. $\mathrm{Br} \mathrm{J}$ Clin Pharmacol 2007; 63(6): 741-52.

[10] Le Meur Y, Büchler M, Thierry A, Caillard S, Villemain F, Lavaud S, Etienne I, Westeel PF, de Ligny BH, Rostaing L, Thervet E, Szelag JC, Rérolle JP, Rousseau A, Touchard G, Marquet P. Individualized mycophenolate mofetil dosing based on drug exposure significantly improves patient outcomes after renal transplantation. Am J Transplant 2007; 7: 2496-2503.

[11] Oellerich M, Shipkova M, Schutz E, Wieland E, Weber LT, Tonshoff B, Armstrong VW. Pharmacokinetic and metabolic investigations of mycophenolic acid in pediatric patients after renal transplantation: implications for therapeutic drug monitoring. German Study Group on Mycophenolate Mofetil Therapy in Pediatric Renal Transplant Recipients. Ther Drug Monit 2000; 22:20-26.

[12] Prémaud A, Debord J, Rousseau A, Le Meur Y, Toupance O, Lebranchu Y, Hoizey G, Le Guellec C, Marquet P. A double absorption-phase model adequately describes mycophenolic acid plasma profiles in de novo renal transplant recipients given oral mycophenolate mofetil. Clin Pharmacokinet 2005; 44(8):837-47.

[13] de Winter BC, van Gelder T, Sombogaard F, Shaw LM, van Hest RM, Mathot RA. Pharmacokinetic role of protein binding of mycophenolic acid and its glucuronide metabolite in renal transplant recipients. J Pharmacokinet Pharmacodyn. 2009 ;36(6):541-64. 
[14] Jiao Z, Ding JJ, Shen J, Liang HQ, Zhong LJ, Wang Y, Zhong MK, Lu WY. Population pharmacokinetic modelling for enterohepatic circulation of mycophenolic acid in healthy Chinese and the influence of polymorphisms in UGT1A9. Br J Clin Pharmacol 2008;65(6):893-907.

[15] Shum B, Duffull SB, Taylor PJ, Tett SE. Population pharmacokinetic analysis of mycophenolic acid in renal transplant recipients following oral administration of mycophenolate mofetil. $\mathrm{Br} \mathrm{J}$ Clin Pharmacol 2003; 56:188-97.

[16] Funaki T. Enterohepatic circulation model for population pharmacokinetic analysis. J Pharm Pharmacol $1999 ; 51(10):$ 1143-8.

[17] Musuamba FT, Rousseau A, Bosmans JL, Senessael JJ, Cumps J, Marquet P, Wallemacq P, Verbeeck RK.Limited sampling models and Bayesian estimation for mycophenolic acid area under the curve prediction in stable renal transplant patients co-medicated with ciclosporin or sirolimus. Clin Pharmacokinet. 2009;48(11):745-58.

[18] Weber LT, Hoecker B, Armstrong VW, Oellerich M, Tönshoff B. Validation of an abbreviated pharmacokinetic profile for the estimation of mycophenolic acid exposure in pediatric renal transplant recipients. Ther Drug Monit 2006; 28: 623-31.

[19] Filler G and Mai I. Limited sampling strategy for mycophenolic acid area under the curve. Ther Drug Monit 2000; 22:169-73.

[20] Schütz E, Armstrong VW, Shipkova M, Weber L, Niedmann PD, Lammersdorf T, Wiesel M, Mandelbaum A, Zimmerhackl LB, Mehls O, Tönshoff B, Oellerich M and Members of the German study group on MMF therapy in pediatric renal transplant recipients. Limited sampling strategy for the determination of mycophenolic acid area under the curve in pediatric kidney recipients. Transplant Proc 1998; 30:1182-4.

[21] Prémaud A, Le Meur Y, Debord J, Szelag JC, Rousseau A, Hoizey G, Toupance O, Marquet P. Maximum a posteriori bayesian estimation of mycophenolic acid pharmacokinetics in renal transplant recipients at different postgrafting periods. Ther Drug Monit. 2005 ; 27(3):354-61. 
[22] Prémaud A, Rousseau A, Le Meur Y, Venisse N, Loichot C, Turcant A, Hoizey G, Compagnon P, Hary L, Debruyne D, Saivin S, Jacqz-Aigrain E, Marquet P. Feasibility of, and critical paths for mycophenolate mofetil Bayesian dose adjustment: pharmacological re-appraisal of a concentrationcontrolled versus fixed-dose trial in renal transplant recipients. Pharmacol Res 2010; 61(2):167-74.

[23] Rousseau A, Laroche ML, Venisse N, Loichot-Roselmac C, Turcant A, Hoizey G, Compagnon P, Hary L, Debruyne D, Saivin S, Jacqz-Aigrain E, Buchler M, Villeneuve C, Vergnenègre A, Le Meur Y, Marquet P. Cost-Effectiveness Analysis of Individualized Mycophenolate Mofetil Dosing in Kidney Transplant Patients in the APOMYGRE Trial. Transplantation 2010 [Epub ahead of print].

[24] Payen S, Zhang D, Maisin A, Popon M, Bensman A, Bouissou F, Loirat C, Gomeni R, Bressolle F, Jacqz-Aigrain E. Population pharmacokinetics of mycophenolic acid in kidney transplant pediatric and adolescent patients. Ther Drug Monit 2005; 27:378-88.

[25] Le Guellec C, Bourgoin H, Büchler M, Le Meur Y, Lebranchu Y, Marquet P, Paintaud G. Population pharmacokinetics and Bayesian estimation of mycophenolic acid concentrations in stable renal transplant patients. Clin Pharmacokinet 2004; 43:253-66.

[26] Staatz CE, Duffull SB, Kiberd B, Fraser AD, Tett SE. Population pharmacokinetics of mycophenolic acid during the first week after renal transplantation. Eur J Clin Pharmacol 2005; 61(7):507-16

[27] van Hest RM, van Gelder T, Vulto AG, Mathot RA. Population pharmacokinetics of mycophenolic acid in renal transplant recipients. Clin Pharmacokinet 2005; 44:1083-96.

[28] de Winter BC, van Gelder T, Glander P, Cattaneo D, Tedesco-Silva H, Neumann I, Hilbrands L, van Hest RM, Pescovitz MD, Budde K, Mathot RA. Population pharmacokinetics of mycophenolic acid: a comparison between enteric-coated mycophenolate sodium and mycophenolate mofetil in renal transplant recipients. Clin Pharmacokinet 2008;47(12): 827-38.

[29] Sam WJ, Akhlaghi F, Rosenbaum SE. Population pharmacokinetics of mycophenolic acid and its 2 glucuronidated metabolites in kidney transplant recipients. J Clin Pharmacol 2009;49(2),: 185-95.

[30] Petersson KJ, Hanze E, Savic RM, Karlsson MO. Semiparametric distributions with estimated shape parameters. Pharm Res 2009; 26(9): 2174-85. 
[31] Leary R, Jelliffe R, Schumitzky A, Van Guilder M. A unified parametric/nonparametric approach to population PK/PD modeling. Annual meeting of the Population Approach Group in Europe, Paris, France, June 6-7, 2002.

[32] Schumitzky A. The nonparametric maximum likelihood approach to pharmacokinetic population analysis. Proceedings of the 1993 Western Simulation Multiconference-Simulation for health care. Society for computer simulation. 1993, pp95-100.

[33] Mallet A. A maximum likelihood estimation method for random coefficient regression moels. Biometrika 1986; 73: 645-56.

[34] Shipkova M, Niedmann PD, Armstrong VW, Schütz E, Wieland E, Shaw LM, Oellerich,M. Simultaneous determination of mycophenolic acid and its glucuronide in human plasma using a simple high-performance liquid chromatography procedure. Clin Chem 1998; 44:1481-8.

[35] Beal SL, Sheiner LB, Boeckmann A. NONMEM user's guides 1989-2006. Icon Development solutions, Ellicott City, MD.

[36] Urien S. Rfn,R for Nonmen. A graphical interface for Nonmen outputs, 2009.

[37] Karlsson MO, Savic RM. Diagnosing model diagnostics. Clin Pharmacol Ther 2007; 82(1):17-20.

[38] DZ D’Argenio and A Schumitzky, ADAPT II User's guide, Pharmacokinetics/Pharmacodynamic Systems Analysis Software, Biomedical Simulations Resource, University of Southern California, 1997.

[39] R Development Core Team. R: A language and environment for statistical computing. R Foundation for Statistical Computing, Vienna, Austria 2009.

[40] Zhao W, Fakhoury M, Deschênes G, Roussey G, Brochard K, Niaudet P, Tsimaratos M, André JL, Cloarec S, Cochat P, Bensman A, Azougagh S, Jacqz-Aigrain E. Population Pharmacokinetics and Pharmacogenetics of Mycophenolic Acid Following Administration of Mycophenolate Mofetil in De Novo Pediatric Renal-Transplant Patients. J Clin Pharmacol 2010; 69(4): 358-66.

[41] Prémaud A, Debord J, Rousseau A, Le Meur Y, Toupance O, Lebranchu Y, Hoizey G, Le Guellec C, Marquet P. A double absorption-phase model adequately describes mycophenolic acid plasma 
profiles in de novo renal transplant recipients given oral mycophenolate mofetil. Clin Pharmacokinet 2005;44(8):837-47.

[42] Hesselink DA, van Hest RM, Mathot RAA, Cyclosporin interacts with mycophenolate mofetil by inhibiting the multidrug resistance-associated protein 2. Am J Transplant 2005;5: 987-94.

[43] Van Gelder T, Klupp J, Barten MJ, Christians U, Morris RE. Comparison of the effects of tacrolimus and cyclosporine on the pharmacokinetics of mycophenolic acid. Ther Drug Monit $2001 ; 23: 119-28$.

[44] Bustad A, Terziivanov D, Leary R, Port R, Schumitzky A, Jelliffe R. Parametric and nonparametric population methods. Their comparative performance in analyzing a clinical dataset and two Monte Carlo simulation studies. Clin Pharmacokinet 2006;45: 365-83. 


\section{FIGURE LEGENDS}

Figure 1: Mycophenolic acid (MPA) concentration-time profiles of patients in the reference and test groups obtained at (a) week $3(n=42)$, (b) month $3(n=47)$, (c) month $6(n=23)$ and (d) month $9(n=17)$ post-transplant.

Figure 2: Scatter plots of (A) observed concentrations (DV) versus population model-predicted concentrations (PRED), (B) observed concentrations (DV) versus individual model-predicted concentrations (IPRED), (C) weighted residuals (WRES) versus model-predicted concentrations (PRED) and (D) weighted residuals (WRES) versus time.

Figure 3: Visual predictive check results of mycophenolic acid (MPA) for (a) early (W3) and (b) stable (M3 and M6) post-transplant periods. Symbols denote observation. Solid line and dashed lines represent the median and the $90 \%$ tolerance interval, respectively. Concentrations are corrected to a mycophenolate mofetil dose of $625 \mathrm{mg}$.

Figure 4: Scatter plot of (A) observed and predicted plasma mycophenolic acid (MPA) concentrations based on the population median parameter values and (B) observed and predicted plasma MPA concentrations based on the median value of each subject's individual nonparametric Bayesian posterior parameter distributions obtained using NonParametric Adaptive Grid (NPAG). 
Table 1. Demographic characteristics of the patients and mycophenolic acid (MPA) dose-normalized $\mathrm{AUC}_{0-12}(\mathrm{mg} . \mathrm{h} / \mathrm{mL}) / \mathrm{mg}$ at the different sampling periods.

\begin{tabular}{|c|c|c|}
\hline & Reference group $(n=34)$ & Test group $(n=32)$ \\
\hline Age (years) $^{a}$ & $11.2 \pm 3.2$ & $8.9 \pm 4.9(\mathrm{NS})$ \\
\hline Bodyweight $(\mathrm{kg})^{\text {a }}$ & $32.2 \pm 11.5$ & $30.5 \pm 16.1(\mathrm{NS})$ \\
\hline Sex ratio $M / F^{a}$ & $23 / 11$ & $19 / 13$ \\
\hline Mycophenolate mofetil dose $(\mathrm{mg})^{a}$ & $625 \pm 178$ & $569 \pm 213(N S)$ \\
\hline \multicolumn{3}{|c|}{ MPA dose-normalized $\mathrm{AUC}_{0-12}(\mathrm{mg} \cdot \mathrm{h} / \mathrm{mL}) / \mathrm{mg}^{\mathrm{b}}$} \\
\hline - All periods & $106(18-415)$ & $91(32-251)$ \\
\hline - Week 3 & $53(18-176)$ & $54(37-122)$ \\
\hline - Month 3 & $104(45-415)$ & $91(42-228)$ \\
\hline - Month 6 & $97(35-183)$ & $124(78-251)$ \\
\hline - Month 9 & $-{ }^{c}$ & $106(32-217)$ \\
\hline \multicolumn{3}{|l|}{${ }^{a}$ data are given as mean $\pm S D$} \\
\hline \multicolumn{3}{|l|}{${ }^{\mathrm{b}}$ data are given as median (range) } \\
\hline \multicolumn{3}{|c|}{${ }^{c}$ reference group did not include data on month 9 post-transplantation } \\
\hline \multicolumn{3}{|c|}{$N S=$ no significant difference with respect to the reference group } \\
\hline
\end{tabular}


Table 2. Mycophenolic acid (MPA) population pharmacokinetic parameters, inter-individual and inter-occasion variabilities, range of individual estimates obtained with the POSTHOC option, and results of the bootstrap internal validation procedure. Bias and precision of Bayesian estimates of MPA exposure in the test group (32 patients, $\mathrm{n}=56$ concentration-time profiles) based on NONMEM population model.

\begin{tabular}{|c|c|c|c|c|c|c|c|}
\hline \multirow[t]{2}{*}{ Parameter } & \multirow{2}{*}{$\begin{array}{l}\text { Final model } \\
\text { original dataset } \\
\text { mean (SE) }\end{array}$} & \multirow{2}{*}{$\begin{array}{l}\text { Range of } \\
\text { individual } \\
\text { estimates }\end{array}$} & \multicolumn{3}{|l|}{ Bootstrap $^{a}$} & \multicolumn{2}{|l|}{ Bayesian forecasting } \\
\hline & & & mean \pm SE & median & $5^{\text {th }}-95^{\text {th }}$ percentiles & & \\
\hline $\begin{array}{l}\text { Fixed effect para } \\
\mathrm{ka}\left(\mathrm{h}^{-1}\right)\end{array}$ & $\begin{array}{l}\text { neters } \\
2.28(0.51)\end{array}$ & $0.22-20.41$ & $2.04 \pm 0.53$ & 1.88 & $1.42-3.06$ & $\begin{array}{l}\text { AUC }_{0-12} \\
\text { Bias, MRE \% }\end{array}$ & $-9.53^{b}$ \\
\hline$V_{c} / F(L)$ & $17.7(1.84)$ & $4.09-64.97$ & $17.31 \pm 2.90$ & 17.10 & $12.60-22.12$ & Precision, RMSE \% & 14.51 \\
\hline $\mathrm{K} 12\left(\mathrm{~h}^{-1}\right)$ & $0.58(0.10)$ & $0.17-1.55$ & $0.60 \pm 0.12$ & 0.57 & $0.46-0.80$ & Range of relative error & $-24.13 \% ;+36.83 \%$ \\
\hline $\begin{array}{l}\text { K21 }\left(h^{-1}\right) \\
\operatorname{ke}\left(h^{-1}\right)\end{array}$ & $\begin{array}{l}0.007(0.001) \\
0.78(0.07)\end{array}$ & $\begin{array}{l}- \\
0.66-1.15\end{array}$ & $\begin{array}{l}0.009 \pm 0.01 \\
0.85 \pm 0.14\end{array}$ & $\begin{array}{l}0.006 \\
0.83\end{array}$ & $\begin{array}{l}0-0.018 \\
0.65-1.10\end{array}$ & Plasma concentrations & \\
\hline Lag-time(h) & $0.26(0.01)$ & $0.21-0.33$ & $0.24 \pm 0.03$ & 0.25 & $0.18-0.28$ & Bias, MRE \% & $13.67^{\mathrm{b}}$ \\
\hline $\begin{array}{l}\text { Inter-individual v } \\
\omega(\mathrm{k} 12)(\%) \\
\omega(\mathrm{ke})(\%) \\
\omega(\text { lag-time) }(\%)\end{array}$ & $\begin{array}{l}\text { riability } \\
62 \text { (Cl } 9511-87) \\
20(\mathrm{Cl} 950-31) \\
15(\mathrm{Cl} 950-23)\end{array}$ & & $\begin{array}{l}62 \pm 13 \\
27 \pm 19 \\
31 \pm 23\end{array}$ & $\begin{array}{l}63 \\
20 \\
18\end{array}$ & $\begin{array}{l}40-80 \\
0-69 \\
0-70\end{array}$ & Precision, RMSE\% & 77 \\
\hline $\begin{array}{l}\text { Inter-occasion vc } \\
\omega(\mathrm{ka})(\%) \\
\omega(\mathrm{Vc} / \mathrm{F})(\%)\end{array}$ & $\begin{array}{l}\text { iability } \\
109 \text { (Cl 95 68-139) } \\
53 \text { (Cl 39-63) }\end{array}$ & & $\begin{array}{l}99 \pm 17 \\
53 \pm 7\end{array}$ & $\begin{array}{l}100 \\
53\end{array}$ & $\begin{array}{l}67-125 \\
42-63\end{array}$ & & \\
\hline $\begin{array}{l}\text { Residual variabil } \\
\sigma_{1}(\%) \\
\sigma_{2}(\mathrm{mg} / \mathrm{L})\end{array}$ & $\begin{array}{l}y \\
38.7 \\
0.08\end{array}$ & & $\begin{array}{l}38 \pm 3 \\
0.11 \pm 0.08\end{array}$ & $\begin{array}{l}38 \\
0.11\end{array}$ & $\begin{array}{l}34-42 \\
0-0.23\end{array}$ & & \\
\hline $\begin{array}{l}\text { ka, absorption con } \\
\text { time. } \\
{ }^{\mathrm{a}} \text { Data from } 1000 \mathrm{k} \\
{ }^{\mathrm{b}} \mathrm{p}<0.05\end{array}$ & $\begin{array}{l}\text { tant; } \mathrm{VC} / \mathrm{F} \text {, apparent } \mathrm{C} \\
\text { otstrap analyses. }\end{array}$ & volume of & ution ; k12 and $k$ & inter-co & nent transfer constar & imination rate constant, lag-ti & e, absorption lag- \\
\hline
\end{tabular}


Table 3. Mycophenolic acid (MPA) population pharmacokinetic parameters obtained in NonParametric Adaptive Grid (NPAG). Bias and precision of Bayesian estimates of MPA exposure in the test group (32 patients, $n=56$ concentration-time profiles) based on NPAG population model.

\section{NPAG population model in reference group}

\begin{tabular}{lllll}
\hline Parameter & Mean & Median & $\begin{array}{l}\text { Inter-individual variability } \\
(\mathrm{CV} \%)\end{array}$ & [Range] \\
\hline $\mathrm{ka}\left(\mathrm{h}^{-1}\right)$ & 5.21 & 2.87 & 92.20 & {$[0.10-20.10]$} \\
$\mathrm{Vc} / \mathrm{F}(\mathrm{L})$ & 16.11 & 12.13 & 74.80 & {$[1.80-56.92]$} \\
$\mathrm{K} 12\left(\mathrm{~h}^{-1}\right)$ & 1.30 & 1.05 & 82.16 & {$[0.002-5.00]$} \\
$\mathrm{K} 21\left(\mathrm{~h}^{-1}\right)$ & 0.69 & 0.03 & 207.83 & {$[0.005-4.99]$} \\
$\mathrm{ke}\left(\mathrm{h}^{-1}\right)$ & 1.16 & 1.05 & 56.40 & {$[0.26-4.33]$} \\
Lag-time $(\mathrm{h})$ & 0.40 & 0.28 & 98.54 & {$[0.0001-1.85]$}
\end{tabular}

\section{Bayesian forecasting in test group}

using NPAG model as PRIORS in ADAPTII

\begin{tabular}{|c|c|c|}
\hline & Normal $^{\mathrm{a}}$ & Lognormal $^{a}$ \\
\hline \multicolumn{3}{|l|}{ AUC0-12 } \\
\hline Bias, MRE \% & 1.40 & -1.68 \\
\hline Precision, RMSE \% & 12.10 & 6.87 \\
\hline Range of relative error & $-11.46 \% ;+65.35 \%$ & $-11.22 \% ;+26.29 \%$ \\
\hline \multicolumn{3}{|l|}{ Plasma concentrations } \\
\hline Bias, MRE \% & 11.53 & 13.6 \\
\hline Precision, RMSE\% & 44.71 & 50.02 \\
\hline
\end{tabular}

50.02

$\mathrm{ka}$, absorption constant; $\mathrm{V} / \mathrm{F}$, apparent central volume of distribution ; $\mathrm{k} 12$ and $\mathrm{k} 21$, inter-compartment transfer constants, ke, elimination rate constant, lag-time, absorption lag-time.

a prior distribution model used in ADAPT II. 
Figure 1
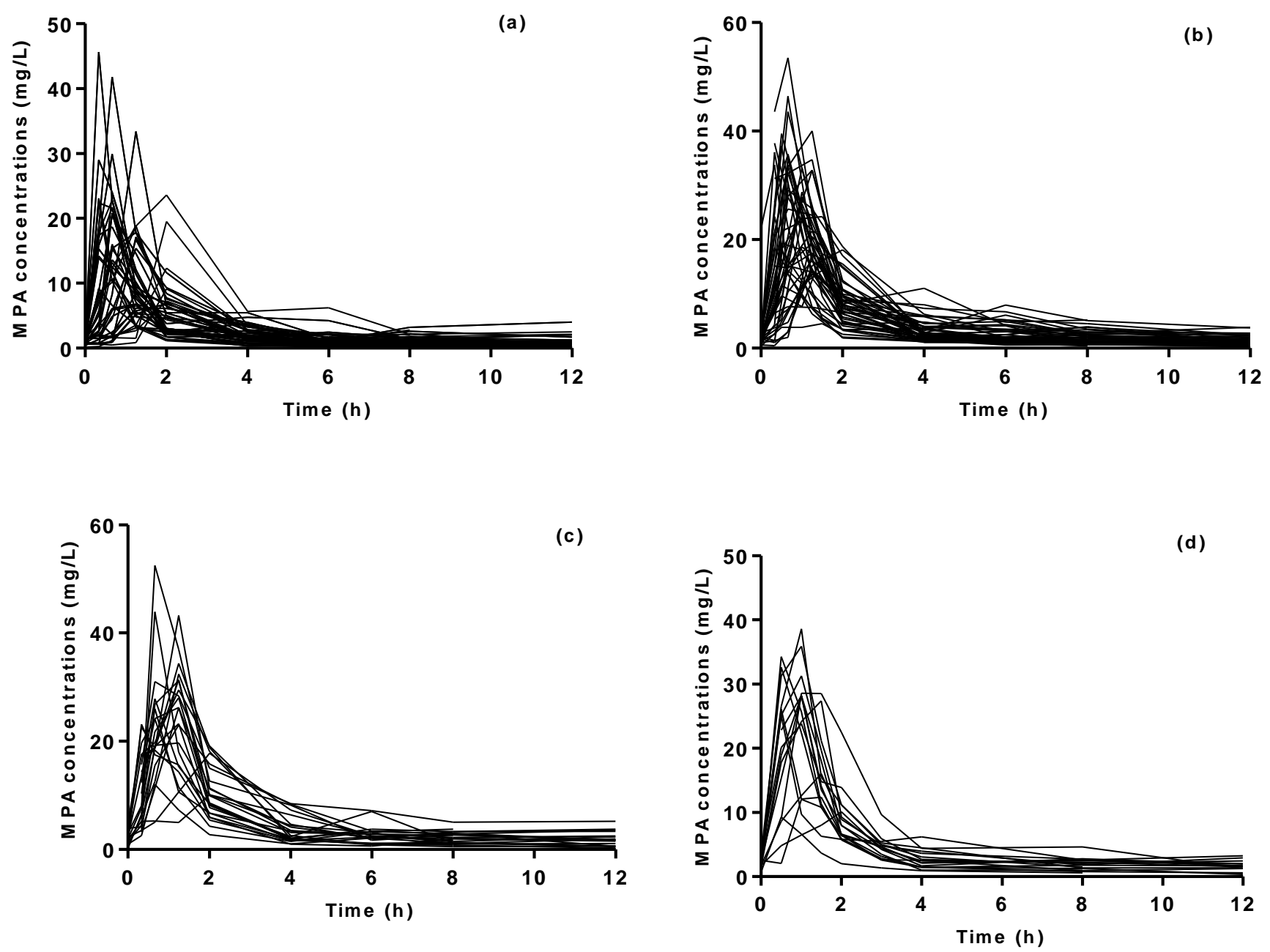
Figure 2
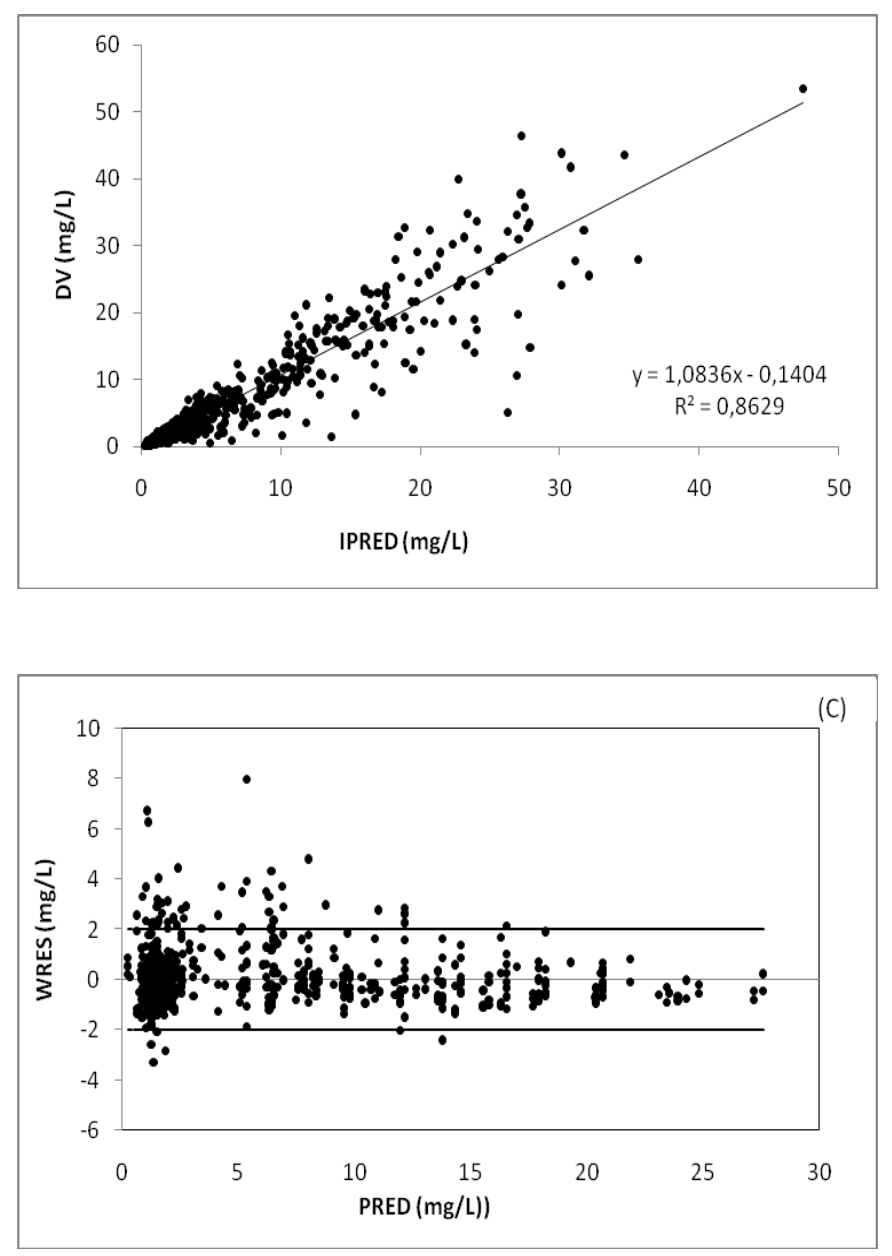
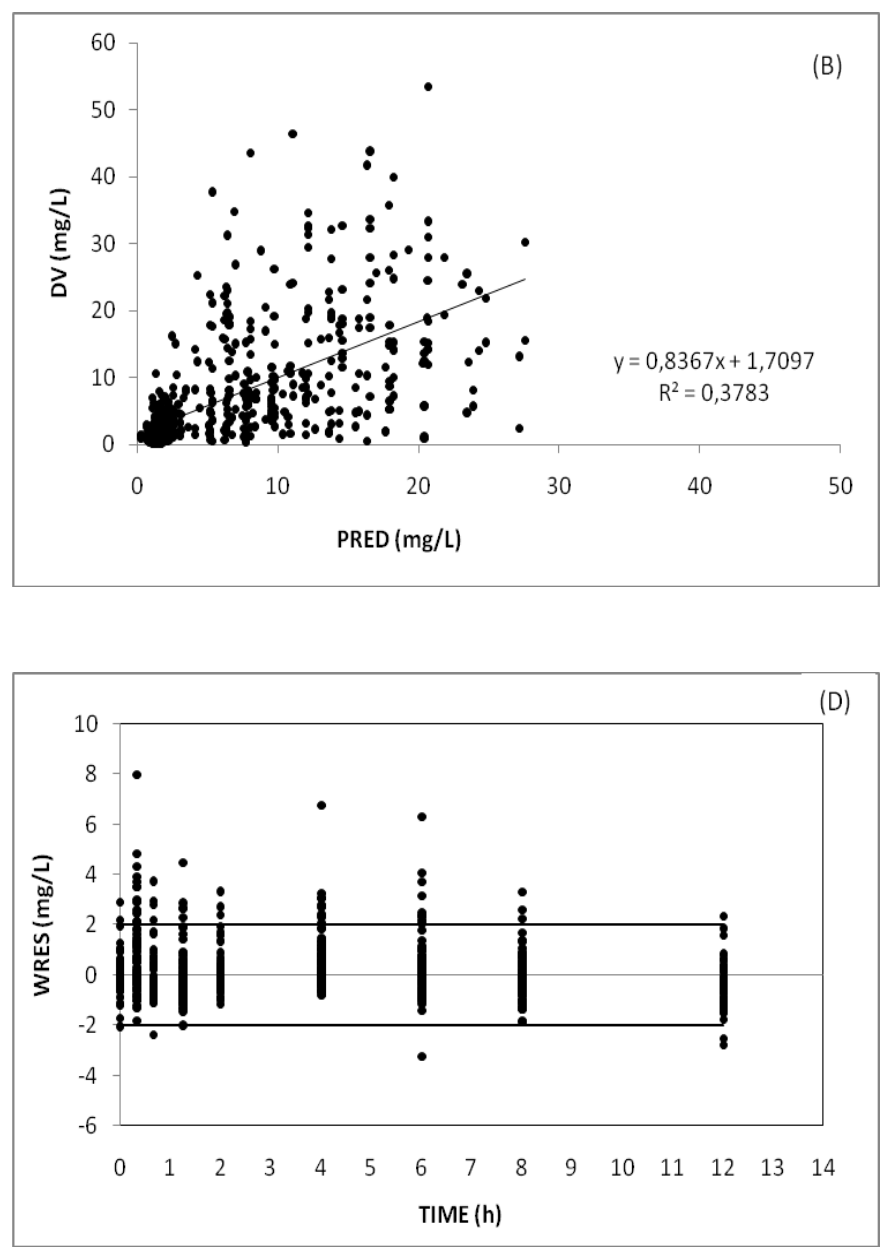

27 
Figure 3
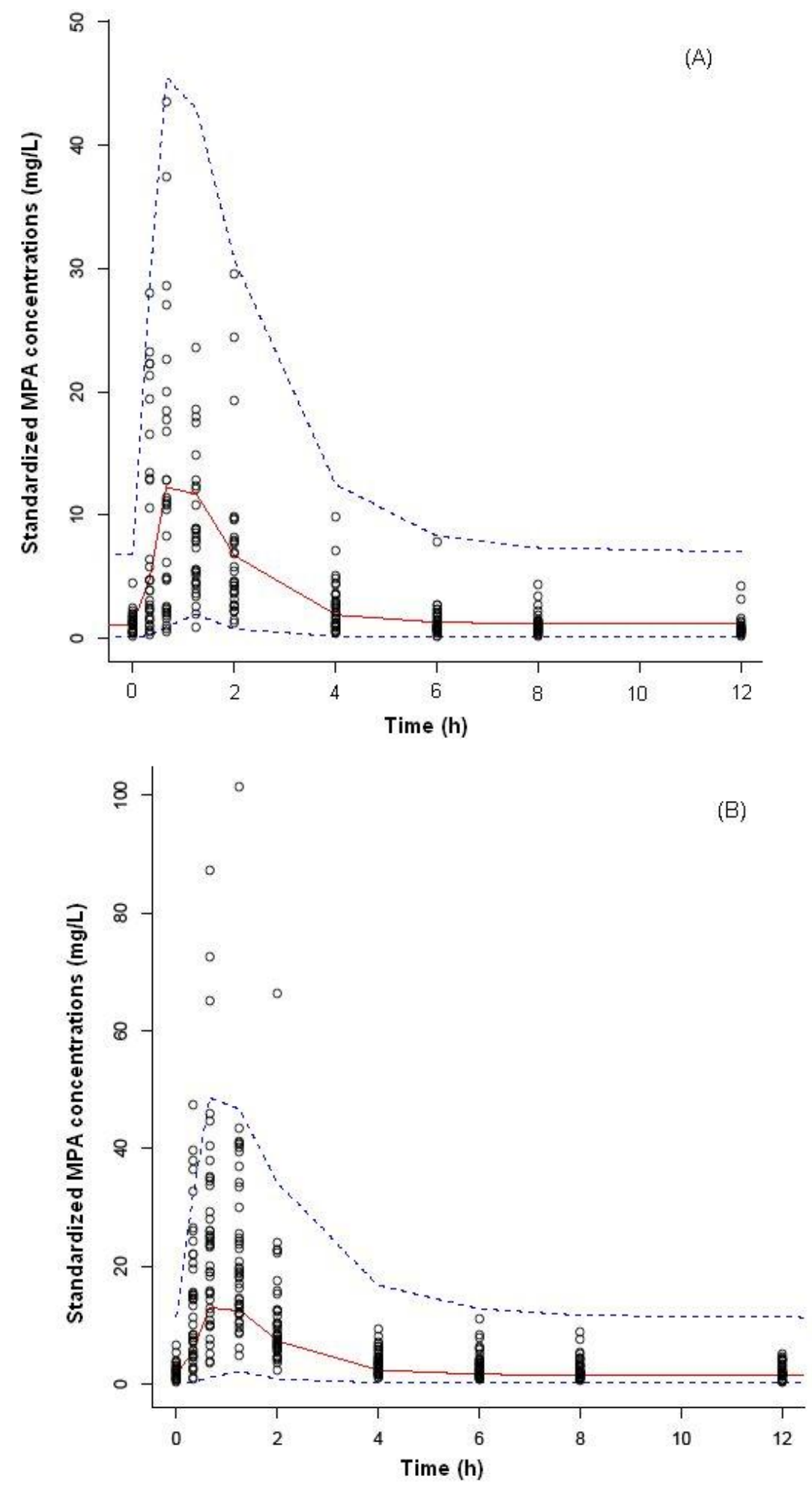
Figure 4

(A)

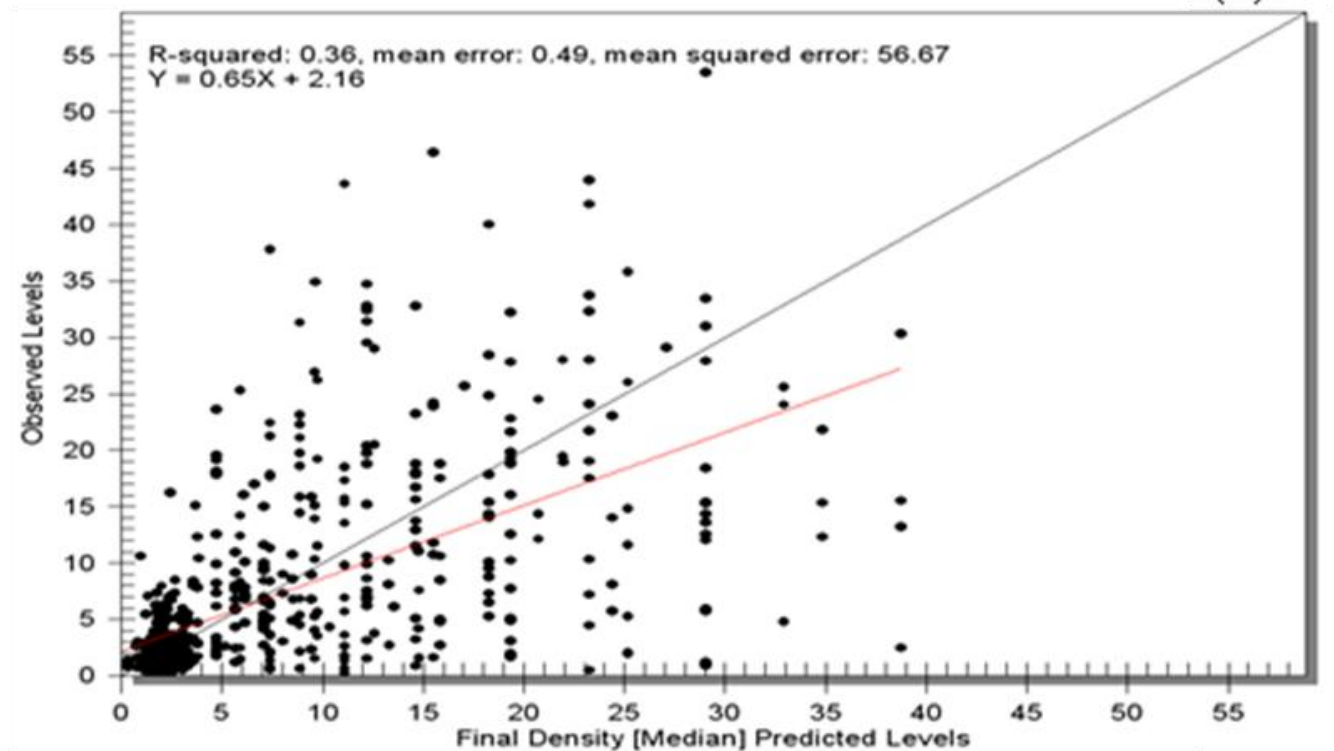

(B)

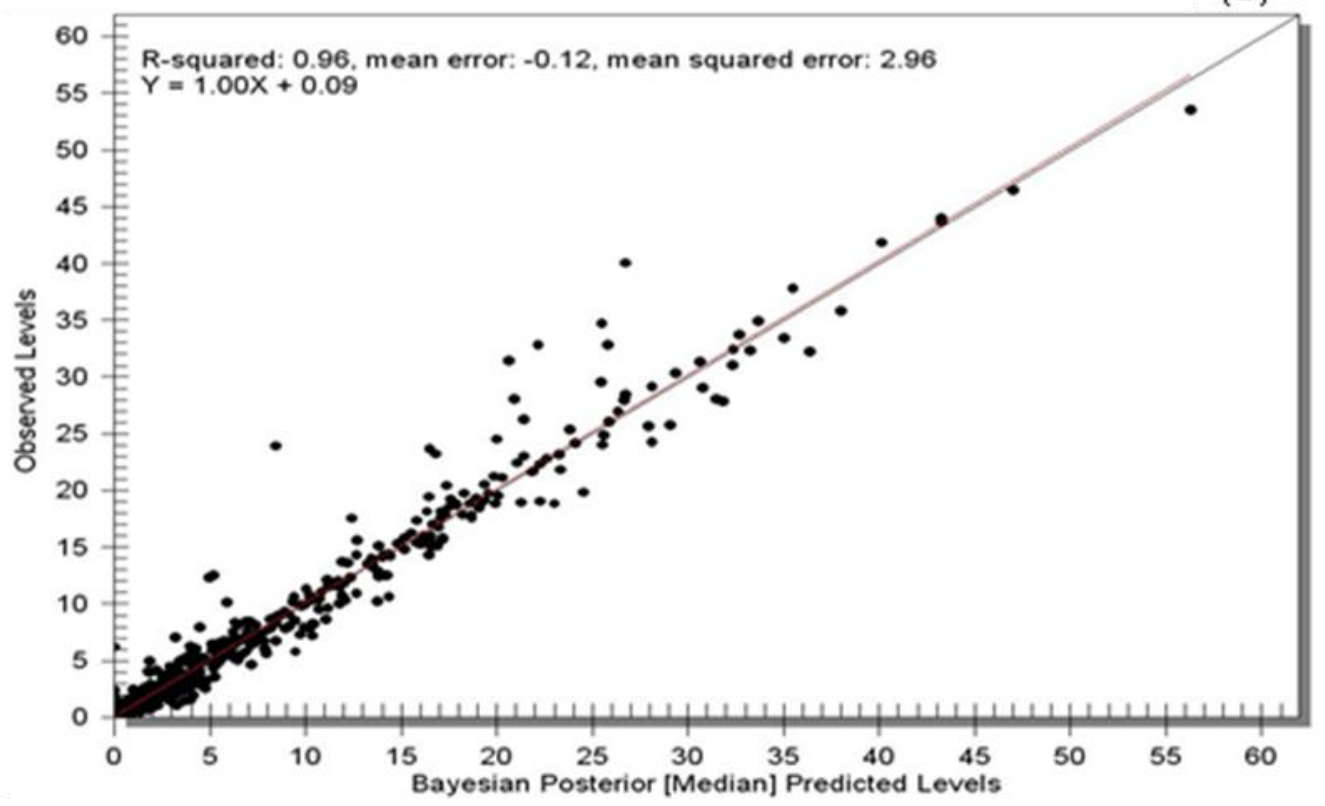


Graphical abstract

MPA pharmacokinetics modeling in pediatric renal transplant recipients

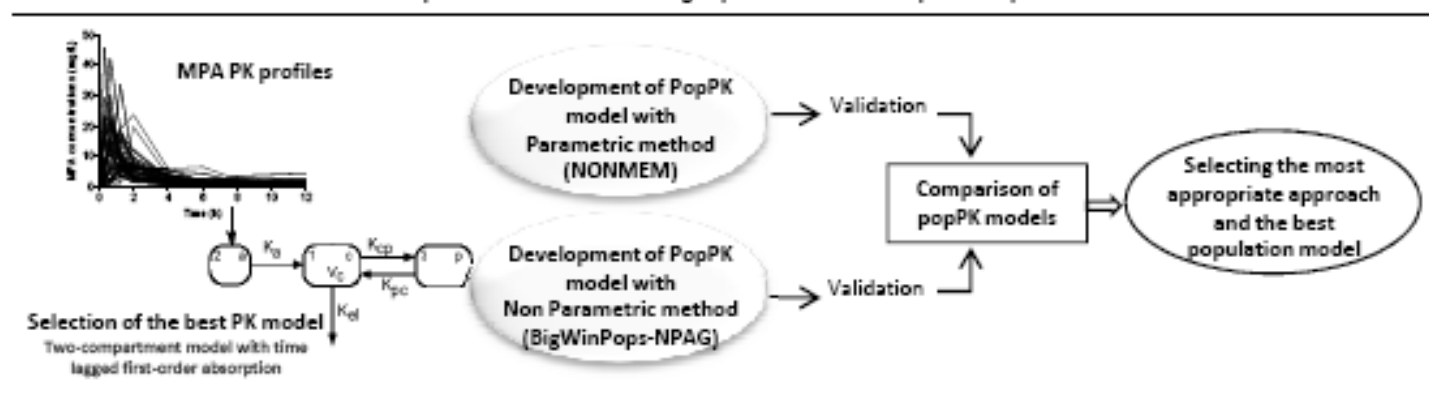

